\title{
Antagonism of NMDA receptors as a potential treatment for Down syndrome: a pilot randomized controlled trial
}

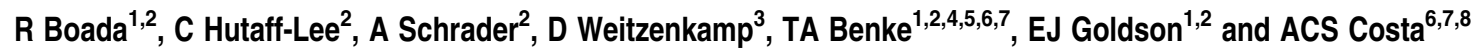

Down syndrome (DS) is the most common genetic cause of intellectual disability. The $N$-methyl-D-aspartate (NMDA) receptor uncompetitive antagonist, memantine hydrochloride (memantine), has been shown to improve learning/memory and rescue one form of hippocampus synaptic plasticity dysfunction in the best-studied mouse model of DS available, the Ts65Dn mouse. Given the status of memantine as a treatment for Alzheimer's disease (AD) approved by the Food and Drug Administration, the preclinical evidence of potential efficacy in Ts65Dn mice, and the favorable safety profile of memantine, we designed a study to investigate whether the findings in the mouse model could be translated to individuals with DS. In this pilot, proof-of-principle study we hypothesized that memantine therapy would improve test scores of young adults with DS on measures of episodic and spatial memory, which are generally considered to be hippocampus dependent. Accordingly, in this randomized, double-blind, placebo-controlled trial, we compared the effect of 16-week treatment with either memantine or placebo on cognitive and adaptive functions of 40 young adults with DS using a carefully selected set of neuropsychological outcome measures. Safety and tolerability were also monitored. Although no significant differences were observed between the memantine and placebo groups on the two primary outcome measures, we found a significant improvement in the memantine group in one of the secondary measures associated with the primary hypothesis. Only infrequent and mild adverse events were noted.

Translational Psychiatry (2012) 2, e141; doi:10.1038/tp.2012.66; published online 17 July 2012

\section{Introduction}

Down syndrome (DS) is caused by the trisomy of chromosome $21,1,2$ and is the most common genetically driven intellectual disability (incidence of 1 in 691 individuals). ${ }^{3}$ Individuals with DS display various degrees of intellectual disability (moderate intellectual disability being the most frequent outcome), deficits in expressive language, syntactic/morphosyntactic processing, verbal working memory, digit span and a disproportionate deficit in hippocampus-dependent function..$^{4-11}$ Neurological phenotypes associated with DS include increased incidence of seizure disorder in relation to the general population, motor and oculomotor dysfunction and a virtually universal incidence of a neuropathology indistinguishable from Alzheimer's disease (AD) by age 40 years. ${ }^{12-15}$ Although the development of clinically detectable cognitive decline in older adults with DS typically lags behind the pathology by 20-25 years, as life expectancy of individuals with DS approaches 60 years, the comorbidity of $D S$ and $A D$ is becoming an increasingly important clinical issue. $^{13}$

Studies on the best-characterized animal model of DS available, the Ts65Dn mouse, have unveiled learning and memory deficits on tests putatively dependent on the functional integrity of the hippocampal formation that may be attributable, at least in part, to excessive signaling via $N$-methyl-D-aspartate (NMDA) receptors. ${ }^{16}$ Some of the evidence for excessive NMDA receptor signaling comes from experiments demonstrating that the uncompetitive antagonist memantine rescues learning and memory deficits in this mouse model of DS, which has now been replicated in three laboratories. ${ }^{16-18}$ Recently, it has been demonstrated that alterations in the form of synaptic plasticity known as longterm depression in the hippocampus of Ts65Dn mice can also be rescued with the use of pharmacologically relevant doses of memantine. ${ }^{19}$

The preclinical evidence of potential efficacy in Ts65Dn mice, ${ }^{16-19}$ coupled to the fact that memantine is a drug approved by the Food and Drug Administration for the treatment of $A D^{19}$ that is well tolerated even in elderly individuals, ${ }^{20,21}$ led us to design a pilot study to investigate whether the findings in the mouse model could be translated to individuals with DS. Accordingly, in a randomized, doubleblind, placebo-controlled trial, we studied the effect of 16-week memantine treatment on cognitive function of young

\footnotetext{
${ }^{1}$ Department of Pediatrics, University of Colorado School of Medicine, Aurora, CO, USA; ${ }^{2}$ The Children's Hospital Colorado, Aurora, CO, USA; ${ }^{3}$ Department of Biostatistics and Informatics, Colorado School of Public Health, University of Colorado Denver, Aurora, CO, USA; ${ }^{4}$ Department of Neurology, University of Colorado School of Medicine, Aurora, CO, USA; ${ }^{5}$ Department of Pharmacology, University of Colorado School of Medicine, Aurora, CO, USA; ${ }^{6}$ Neuroscience Training Program, University of Colorado Denver, Aurora, CO, USA; ${ }^{7}$ Colorado Intellectual and Developmental Disability Research Center, University of Colorado Denver, Aurora, CO, USA and ${ }^{8}$ Department of Medicine, University of Colorado School of Medicine, Aurora, CO, USA

Correspondence: Dr ACS Costa, Department of Medicine, Division of Clinical Pharmacology and Toxicology, University of Colorado School of Medicine, 12700 East 19 th Avenue, MS C-237, Aurora, CO 80045, USA.

E-mail: Alberto.Costa@ucdenver.edu

Keywords: Alzheimer disease; Down syndrome; intellectual disability; memantine; trisomy 21; Ts65Dn

Received 24 April 2012; revised 11 June 2012; accepted 14 June 2012
} 
adults with DS using neuropsychological outcome measures. Specifically, in this pilot, proof-of-principle study we hypothesized that memantine therapy might improve test scores of young adults with DS on measures of episodic and spatial memory, which are generally considered to be hippocampus dependent.

\section{Subjects and methods}

Design. A prospective, double-blind, placebo-controlled, randomized, single-site, 16-week clinical trial was conducted in young adults with DS. This study was conducted in a matched-pair design. The protocol was approved by the Colorado Multiple Institutional Review Board, University of Colorado Anschutz Medical Campus, CO, USA (ClinicalTrials.gov identifier: NCT01112683).

Participants. A total of 42 individuals with DS from both genders and between the ages of 18 and 32 years were recruited from the community. A total of 39 participants had a cytogenetic diagnostic of trisomy 21 and 3 participants had complete unbalanced Robertsonian translocations involving a 14 and 21 homolog, leading to an additional chromosome 21. All participants were enrolled by the senior author (ACSC) who performed a screening interview, interactively explained the goals and procedures involved in the study, and obtained written informed consent (participants and parents/legal guardians) and assent (participants). Participants were subsequently seen by either a pediatrician or child neurologist (EJG or TAB) for a baseline medical assessment and confirmation of conformity with inclusion and exclusion criteria. There was no specific cognitive level used to exclude participants; instead, the senior author made a clinical determination regarding each participant's ability to cope with the demands of the study (for example, by observing the ability of the participants to sit still during the screening interview, by asking the participants a few simple questions, including questions regarding their overall understanding of the goals of the trial, and by asking the opinion of the parent or guardian about the participant's ability to undergo the procedures involved in the trial). No participant was excluded because of an inability to complete the neuropsychological test battery. At all times, dissent to participate in the study was assessed by research staff.

Inclusion/exclusion criteria. Inclusion criteria were documented cytogenetic diagnostic of trisomy 21 or translocation DS; age 18 to 32 years; female participants were documented not to be pregnant by serum testing at clinical screening and all subsequent medical visits; participants were required to be in generally good health; laboratory findings had to be within normal limits or judged clinically insignificant at baseline; vital signs must have been within normal limits for their age; screening electrocardiogram had to demonstrate predominately normal sinus rhythm (minor abnormalities documented as clinically insignificant by the investigators and a cardiologist were allowed); participants had to be sufficiently proficient in
English to be capable of reliably completing study assessments; participants had to be able to swallow oral medication (crushing of tablets was not permitted) and have a reliable caregiver or family member who agreed to accompany her/him to all visits, provide information about the participant as required by the protocol, and ensure compliance with the medication schedule; and finally, all participants were required to have contact at least once a day with the responsible caregiver or family member.

Exclusion criteria were weight $<40 \mathrm{~kg}$; any active psychiatric or neurologic diagnosis other than DS; participants who had met the DSM-IV (Diagnostic and Statistical Manual of Mental Disorders-Fourth Edition) criteria for drug or alcohol abuse or dependence within the past 5 years; participants who, in the judgment of the investigators, represented a significant suicide risk or who required treatment with electroconvulsive therapy or with psychotropic drugs during the study or who received treatment with a depot neuroleptic drug within 6 months of entering the study; participants who had been hospitalized or had been residing in a skilled nursing facility or participants who were anticipated to enter a nursing home within 6 months after starting the study protocol; any active or clinically significant conditions affecting absorption, distribution or metabolism of the study drug (for example, inflammatory bowel disease, celiac disease, gastric or duodenal ulcers); participants with significant allergies to or other significant intolerance to memantine therapy, its ingredients, or with contraindications to memantine therapy as stated in the prescribing information; participants who were expected to require general anesthetics during the course of the study; history or presence of seizure disorder ( $<3$ years) or encephalitis; history of malignant neoplasms treated within 3 years before study entry or where there was evidence of recurrent or metastatic disease; participants with treated hypothyroidism were required to be on a stable dose of medication for at least 3 months before screening and have normal serum free T4 and thyroid-stimulating hormone (TSH) levels at screening; participants with diabetes mellitus controlled by diet, oral medication or insulin were required to have a glycated hemoglobin $(\mathrm{HbA} 1 \mathrm{c})$ of $<8.0 \%$ and random serum glucose value of $<170 \mathrm{mg} \mathrm{dl}^{-1}$; history of severe infections or a major surgical operation within 3 months before screening; history of persistent cognitive deficits immediately following head trauma; participants were required not to donate blood or blood products during the 30 days before screening or donate blood while participating in the study or within 4 weeks after completion of the study; participants who were judged not able to comply with the protocol or perform the outcomes measures because of significant hearing or visual impairment or other issues judged relevant by the investigators. Participants and caregiver or family member were asked whether the participant had been receiving any experimental drug or supplement for DS, in which case they would have to undergo a washout $(\sim 30$ days or five half-lives of the drug, whichever was longer); however, none of our study participants were receiving any experimental drug or supplement for DS. Gross assessments of vision and hearing were performed by the clinicians to avoid any potential misinterpretation of neuropsychological assessments due to vision and hearing deficits. 
Protocol. The trial was conducted in a double blind fashion; participants were randomized to memantine or placebo by the Children's Hospital Colorado research pharmacist once initial medical and laboratory screenings were conducted. After a participant passed the initial medical and laboratory screenings and was deemed acceptable to participate in the trial, he or she was matched with another participant on gender and age. One participant from each pair was randomly assigned to receive memantine treatment and the other was assigned to the placebo group. In this manner, the ratio of participants in the memantine versus placebo conditions was 1:1. Participants, caregivers and all other investigators were blind to the treatment allocation.

The drug dosage followed standard titration schedule of memantine for the treatment of $A D$ (that is, $5 \mathrm{mg}$ once daily on week $1,5 \mathrm{mg}$ twice daily on week 2,5 and $10 \mathrm{mg}_{\text {day }}{ }^{-1}$ divided dose on week 3 and $10 \mathrm{mg}$ twice daily from week 4 to week 16). Both the memantine hydrochloride (memantine) and placebo tablets (containing the same fillers and binders as the memantine tablets, but no active ingredients) were filmcoated, white in color and visually indistinguishable in terms of shape and size. Caregivers took responsibility for overseeing the administration of the tablets to the participants. Medication compliance was assessed at a follow-up medical visit (with either EJG or TAB) 8 weeks after the first dose was taken and a second and final medical visit (also with either EJG or TAB). At each medical visit, the medication bottle(s) from the previous treatment period was to be returned to the investigator. The medication was inventoried and the percent compliance calculated by dividing the number of daily doses removed from the bottle by the number of days of the treatment period.

Participants underwent a baseline neuropsychological testing before being given any study medication, and another at the end of the 16-week trial. Testing was administered at the same time of day for every participant $(1000 \mathrm{~h} \pm 30 \mathrm{~min})$, and in the same suite of testing rooms. One board-certified clinical neuropsychologist (RB) and two graduate research assistants experienced in the examination of individuals with intellectual disability ( $\mathrm{CH}-\mathrm{L}$ and $\mathrm{AS}$; directly supervised by $\mathrm{RB}$ ) tested all of the participants. The same tester examined the participant at baseline and at 16 weeks.

At each visit, the participant and their parent/guardian (henceforth, caregiver) entered the testing room and engaged in casual conversation with the examiner for a few minutes until the participant felt comfortable with the situation. Participants were explained the nature of the evaluation in simple terms, and were told that no aspect of it would be painful. The caregiver remained in the room during the first two tasks administered, the second being the CVLT-II (California Verbal Learning Test-II). The caregiver sat in a corner of the room and did not engage with the participant during this time. The caregiver provided verification of verbal responses in case the participant's speech intelligibility was poor on an as-needed basis. Verbal responses during the CVLT-II were also tape recorded for later accuracy checks. The caregiver then left the room and completed an adaptive functioning questionnaire (Scales of Independent Behavior-Revised (SIB-R)) during the remainder of the 2 -h testing period. Participants were given breaks as needed. All participants completed the test battery, with some needing increased prompting and verbal reinforcement to remain on task. The full version of the CVLT-II was administered to the first participant of the trial, and it became clear that the number of items was too great to obtain reliable data. The short form of the CVLT-II (which has 9 items instead of 16) was substituted from the second participant onward, and thus, the first participant has missing data for this particular test. The battery of tests was identical across the two time points. Alternate versions were only available for the computerized measures from the CANTAB (Cambridge Neuropsychological Test Automated Battery). Scoring of all of the tests was conducted by the tester and supervised by a licensed neuropsychologist (RB).

Outcome measures. The battery chosen for this trial mirrors closely that in the work of Pennington et al., ${ }^{7}$ which carefully examined the neuropsychological profile of children with DS. The current battery included assessment of skills in five domains: intellectual functioning; language and vocabulary; visual and verbal episodic memory; visual and verbal working memory; and adaptive/behavioral functioning. The list of tests used in the present study (and their respective abbreviations), grouped by cognitive domain, is provided in Table 1.

Given the mechanism of action of memantine and the hypothesized effects on hippocampal functioning in particular, two primary measures of episodic memory were chosen for this study: Paired Associate Learning (PAL) and Pattern Recognition Memory (PRM). Both of these tasks showed significant differences between participants with DS and mental-age-matched controls in the study by Pennington et al. $^{7}$ Both of these tasks comprise nonverbal stimuli, are administered by computer and require only a motor response (pointing on a touch screen). Therefore, they were thought to be the best candidates to assess memory outcome in this cohort of participants with DS. Due to the exploratory nature of this study, two additional secondary measures of hippocampal function were also administered, one assessing episodic

Table 1 Neuropsychological measures by cognitive domain

Cognitive and adaptive functioning

Scales of Independent Behavior-Revised (SIB-R)

Differential Ability Scales-II (DAS II) Matrices

Hippocampal measures (all require long-term memory)

CANTAB Paired Associate Learning (PAL) ${ }^{a}$

CANTAB Pattern Recognition Memory (PRM) ${ }^{a}$

California Verbal Learning Test-II (CVLT-II) short form ${ }^{b}$

Rivermead Behavioral Memory Test-Children's version (RBMT) ${ }^{\mathrm{b}}$

Prefrontal measures (all require working memory)

CANTAB Spatial Working Memory (SWM)

DAS-II Recall of Digits

\section{Language measures \\ Peabody Picture Vocabulary Test-3rd edition (PPVT-III) \\ Test of Reception of Grammar-2nd edition (TROG-II)}

DAS-II Verbal Fluency

Abbreviation: CANTAB, Cambridge Neuropsychological Test Automated Battery.

aprimary memory outcome measures. becondary memory outcome measures associated with the primary hypothesis. Language and prefrontal measures are secondary discriminant measures not expected to change with treatment. 
verbal memory (CVLT-II) and the other being a more ecological assessment of memory (Rivermead Behavioral Memory Test-Children's version (RBMT)). Only three subtests of the RBMT were used (that is, Route Learning, Message and Belonging tasks). The rest of the measures included in the battery assessed components of neurocognitive functioning that were not predicted to change because of treatment with memantine. Hence, they are deemed to be discriminant measures in this trial, to help assess whether any positive effects of memantine are specific. As shown in Table 1, measures of nonverbal reasoning, receptive language and vocabulary, short-term phonological memory, verbal and nonverbal working memory and adaptive/behavioral functioning were included.

All the measures used in this battery were selected with the mental age of the participants in mind, rather than their chronological age. Because assessing differential change over time was the main goal, tests that were likely not to have basal or ceiling effects for this cohort were selected. For most tests, this means that age norms were not applicable. Raw scores were used as dependent variables in almost all cases, with the exception of the DAS-II (Differential Ability Scales-II) Matrices and SIB-R tests. Because of the nature of the DAS-II Matrices subtest, where participants can be administered different sets of items based on basal and ceiling rules, an intermediate 'Ability Score' derived from the Rasch scaling methodology of the test was used. This allows comparison among participants, but is not a developmental quotient or standard score. For the SIB-R, standard scores were used as age norms were available, thus allowing for at least one direct comparison of adaptive functioning relative to same age peers (see Supplementary Materials for a brief description of each neuropsychological test used in the present study.)

Safety and tolerability were monitored by physical examinations, electrocardiograms, clinical laboratory tests and incidence of adverse event recording. The clinical laboratory tests included assessments of liver function (bilirubin, alkaline phosphatase, alanine amino transferase and aspartate amino transferase) and kidney function (blood urea nitrogen and creatinine), electrolytes (sodium, potassium, calcium, chloride, bicarbonate), blood glucose and proteins (albumin, total protein and hemoglobin), including hemoglobin A1C (at baseline for all participants, and at all visits for those with controlled diabetes), and thyroid group (T4 total, T4 free and $\mathrm{TSH}$ ) at baseline and serum TSH at treatment week 16. This clinical trial involved two clinical laboratory tests per participant (baseline and treatment week 16). Electrocardiograms , with interpretation by Children's Cardiology Service, involved two 12-lead electrocardiograms per participant. Clinical consultations were performed either by a board-certified child neurologist (TB) or a board-certified pediatrician (EJG). The use of pediatricians in this pilot study can be justified by the ultimate goal of this research, which is to extend the use of memantine to the pediatric population, in which we are more likely to have a larger impact on improving the quality of life of individuals with DS. In addition, historically, pediatricians have had extensive experience in diagnosing and treating children with intellectual disabilities into adulthood. Finally, the neuropsychology team at the Children's Hospital Colorado has extensive experience in assessing the cognitive abilities of individuals of comparable mental age. The physical exam included a health questionnaire readdressing the study inclusion/exclusion criteria. In a checklist format, aspects of the general and neurological exam were documented.

Statistical analyses. The various hypotheses generated before data collection for this trial dictated the order and priority of analyses. Sample characteristics were reported first, followed by the main analyses of the primary and secondary outcome measures related to memory functioning. Group differences in discriminant secondary measures were reported next. Analyses describing the relations among outcome measures, with age as a coviariate, were reported last. Given the matched-pair design of the study, this characteristic was incorporated in the analytic techniques as described below. As aforementioned, published studies on the mouse model of DS Ts65Dn have shown convincing evidence of NMDA receptor dysfunction and of preclinical efficacy of memantine in enhancing learning and memory, as well as reversing one type of hippocampal synaptic plasticity alteration. ${ }^{22,15-17}$ These studies led to specific a priori hypotheses regarding the direction of effects. Therefore, one-tailed tests were conducted for all primary and secondary outcome variable analyses. An $\alpha$ level of 0.05 was selected as the criterion for statistical significance, but given the exploratory nature of the current trial, no correction was made for multiple comparisons, so as to minimize type II errors. Effect sizes are also reported, given the small sample size. All statistical analyses were completed in SAS 9.3 (SAS Institute, Cary, NC, USA) or SPSS 19.0 (IBM Corporation, Somers, NY, USA).

The sample size for this project was originally calculated from power analyses performed on the data from Pennington et al. $^{7}$ on various neuropsychological measures on a similar population of individuals with DS. A total of 20 subjects per treatment group was expected to provide $\sim 60 \%$ power to detect a between-group mean difference of 1.2 patterns placed correctly on the PAL (first trial) with respect to change from baseline to treatment week 16. This sample size was also expected to provide $40 \%$ power to detect a betweengroup mean difference of 1.2 patterns recognized on the PRM with respect to change from baseline to week 16. (These numbers were based on the mean differences in these values found between the group of participants with DS and a control group of mental-age-matched typically developing individuals in Table 5 of the original work by Pennington et al. ${ }^{7}$ ) A twosided test at a type I error rate of $5 \%$ was used. This sample size had incorporated an inflation factor of $20 \%$ to account for the ineligibility of an estimated $10 \%$ of randomized participants. At the time this study was designed, the work by Pennington et al. ${ }^{7}$ was the only reference available for the use of the PAL and PRM in a group of individuals with DS and, therefore, we did not benefit from the later expansion of this work by Edgin et al. ${ }^{10}$

For the main analyses looking at primary and secondary outcome measures, repeated measures mixed models were tested using the MIXED analytic program available in SAS. MIXED models more appropriately handle correlated data, missing data and unequal variances, all of which are common in similar longitudinal experiments with neuropsychological 
data. Primary analyses tested for a significant interaction between medication status (drug versus placebo) and time of visit (baseline versus 16-week outcome testing), taking into account the matched-pair design. Group differences at baseline were also assessed using MIXED, in order to ensure that matching and randomization to treatment equalized the two groups on all of the outcome measures of interest at baseline. Effect sizes for all cognitive and adaptive functioning variables were computed by Cohen's $d$, using pooled means and s.d. derived from MIXED through the following formula:

$$
d=\frac{\mu_{m}-\mu_{p}}{\sqrt{\frac{\left(n_{m}-1\right) \cdot s_{m}^{2}+\left(n_{p}-1\right) \cdot s_{p}^{2}}{n_{m}+n_{p}-2}}}
$$

Where $\mu_{m}$ and $\mu_{p}$ are the estimated mean differences in the value of the measure at week 16 of the trial (T2) minus its value before exposure to the study medication (T1), for the memantine and placebo groups, respectively; $n_{m}$ and $n_{p}$ are the numbers of participants in the memantine and placebo group; $s_{m}^{2}$ and $s_{p}^{2}$ represent the s.d. of the differences in the value of the measure at T2 minus its value at T1 for the memantine and placebo groups.

Finally, to examine the relations among outcome variables, Pearson's $r$ correlations for each time point were computed across the whole sample. Point biserial correlation was used to explore the relationship between the outcome variables and gender.

\section{Results}

Recruitment. From the 42 participants recruited, 38 participants completed neuropsychological testing at both baseline and 16 weeks. Two participants who signed the consent form and underwent the baseline medical assessment declined to continue their participation before the beginning of the treatment: the first was because of an unrelated medical issue (cataracts surgery followed by complications) and the second because of personal reasons (death in the family, which, unfortunately, happened after randomization). Additionally, we could not find an age and gender matching pair for one of the participants. One participant dropped out of the study because of parent complaints of increased anxiety, and another was excluded from analyses because of side effects (increased and persistent anxiety) reported at study completion. Data from the latter two participants were included in the sample description analyses as well as the analyses regarding safety. The overall recruitment and inclusion flow into the study is outlined in Figure 1.

There were no differences expected between the placebo and memantine groups on age or gender, given the matchedpair design. In each group, $62 \%$ of the participants were female. The mean age of the participants in the placebo group (22 years and 7 months, range 18-31) did not differ from the mean age of the participants in the memantine group (23 years and 3 months; range 18-30; $t(36)=-0.552$, $P=0.59$ ). Parent's years of education was used to index the

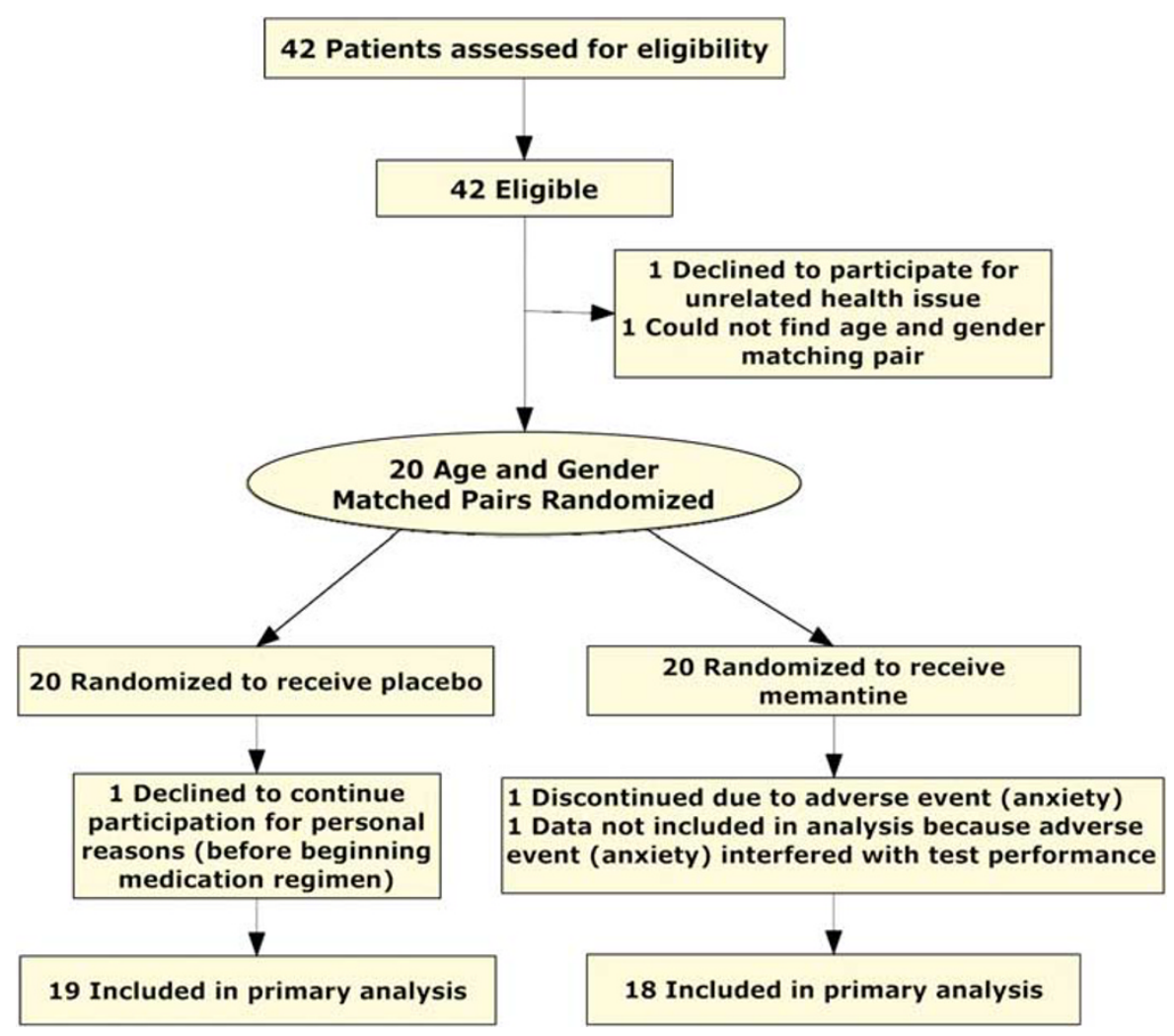

Figure 1 Flowchart of patient inclusion. 
socioeconomic status. There were no significant differences between the placebo and memantine groups on the years of education of the mothers or fathers (mother: $t(36)=1.43$, $P=0.16$; father: $t(36)=-0.43, P=0.67)$.

In terms of common DS comorbidities, the two most frequent conditions seen in our cohort were hypothyroidism (42.1\%) and obesity (31.6\%). (As per inclusion/exclusion criteria, participants with treated hypothyroidism were required to be on a stable dose of medication for at least 3 months before screening and have normal serum free T4 and TSH levels at screening.) Signs and symptoms suggestive of sleep apnea were reported by the parents of $18.4 \%$ of the participants and well-controlled diabetes type I was seen in 2 participants $(5.3 \%)$. No significant statistical differences were found between the memantine and placebo groups in terms of incidence of any of these comorbid medical conditions. With the exception of three participants, oral levothyroxine (to treat hypothyroidism), insulin and birth control pills were the only concomitant medications taken by the study participants. Of the three participants who were taking concomitant medications other than these, we had one participant who was taking sertraline (to control anxiety for more than 5 years), one participant who was taking methylphenidate (to treat signs and symptoms compatible with attention deficit hyperactivity disorder for more than 5 years) and one participant who was taking lamotrigine (to control seizure disorder for $\sim 4$ years, with last spell more than 3 years before enrolling in this study).

The mean intellectual quotient (IQ) of adolescents and young adults with DS is in the low-to-mid 40s. ${ }^{4-7}$ The average mental ages of the study participants on the PPVT-III were 6.1 years for those in the placebo group, and 7.6 years for those in the memantine group. Given that the PPVT-III correlates with the Wechsler series Full Scale IQ at $\sim r=0.85,{ }^{23}$ the cognitive abilities of the current sample are consistent with the literature. As can be seen in Table 2, at baseline, the groups did not differ on the PPVT-III standard score $(t(36)=-1.22$, $P=0.23)$. Last, the groups were also equivalent on the Broad Independence standard score of the SIB-R $(t(32)=-0.23$,
$P=0.82$ ). (See Table 2 for a summary of the characteristics and comparisons between the memantine and placebo groups.)

Effect of memantine on outcome measures. For the main analyses, we ran mixed effects models, with visit (baseline versus 16 weeks) as the within-subject variable and medication status (placebo versus memantine) as the between-subject variable. Each primary and secondary cognitive and adaptive functioning variable was independently tested as the dependent measure. A contrast effect, based on our a priori hypothesis, tested whether the participants in the memantine group had a disproportionate improvement in test scores over the 16week interval compared with the participants in the placebo group. Specifically, the contrast effect tested whether the pre/post difference for each outcome variable was larger in the memantine group than in the placebo group. The Akaike Information Criterion fit statistic was used to decide that it was more appropriate to model correlations between time points only within an individual, and not across participants in a pair. (Means and s.d. for all the dependent variables analyzed are shown in Supplementary Table).

Results of the MIXED model repeated measures analyses for the two primary measures (Table 3 ) only detected a $P$ value of $<0.10$ for the PAL stages score $(F(1,34)=1.90$, $P=0.088)$; the mean pre/post difference for the memantine group was $0.56(90 \%$ confidence interval $(\mathrm{Cl})=-0.07$ to $1.04)$, whereas it was $0.00(90 \%$ confidence interval $=-0.48$ to 0.48 ) for the placebo group. Additionally, significant contrast effect was found for one secondary measure of memory functioning, the CVLT-II Free Recall Total score $\left(F(1,32)=3.02,{ }^{\star} P=0.046\right)$. Pre/post difference scores on the CVLT-II Free Recall Total Score were $5.84(90 \%$ $\mathrm{Cl}=3.47-8.21)$ for the memantine group and $2.53(90 \%$ $\mathrm{Cl}=0.33-4.72)$ for the placebo group, showing that the memantine group scored disproportionately better than the placebo group at 16 weeks. (A visual representation of the raw data and the CVLT-II Free Recall Total Score analysis

Table 2 Baseline characteristics of the study groups

\begin{tabular}{|c|c|c|c|c|}
\hline & Memantine $(\mathrm{N}=19)$ & Placebo $(\mathbf{N}=19)$ & $\mathrm{t}$-Value or $\chi^{2}$ & P-value \\
\hline Age at time of randomization mean \pm s.d. (in months) & $279.26 \pm 42.2$ & $271.16 \pm 48.1$ & -0.55 & 0.59 \\
\hline Gender (\% female) & 63.2 & 63.2 & $0^{a}$ & 1.0 \\
\hline Mother years of education mean \pm s.d. (in years) & $15.37 \pm 2.5$ & $16.53 \pm 2.5$ & 1.43 & 0.16 \\
\hline Father years of education mean \pm s.d. (in years) & $16.00 \pm 2.4$ & $15.58 \pm 3.6$ & -0.43 & 0.67 \\
\hline Hypothyroidism $^{\mathrm{b}}(\%)$ & 36.8 & 47.4 & 0.43 & 0.51 \\
\hline Obesity $(\%)$ & 36.8 & 26.3 & 0.49 & 0.49 \\
\hline Sleep apnead (\%) & 21.1 & 15.8 & 0.18 & 0.68 \\
\hline Diabetes (\%) & 5.3 & 5.3 & $1.0^{\mathrm{e}}$ & 0.76 \\
\hline $\begin{array}{l}\text { Adaptive Functioning mean } \pm \text { s.d. (SIB-R Broad Independence } \\
\text { standard score) }\end{array}$ & $37.41 \pm 14.6$ & $36.00 \pm 20.7$ & -0.23 & 0.82 \\
\hline Nonverbal reasoning mean \pm s.d. (DAS-II Matrices Ability score) ${ }^{f}$ & $55.7 \pm 17.5$ & $51.21 \pm 9.3$ & -0.99 & 0.33 \\
\hline Receptive vocabulary mean \pm s.d. (PPVT-III standard score) & $53.74 \pm 19.3$ & $47.26 \pm 12.9$ & -1.22 & 0.23 \\
\hline
\end{tabular}

Abbreviations: DAS-II, Differential Ability Scales-II; PPVT-III, Peabody Picture Vocabulary Test-3rd edition; SIB-R, Scales of Independent Behavior-Revised.

${ }^{a}$ The $\chi^{2}$ value is 0 as placebo-control pairs were matched on gender, making proportions identical. ${ }^{\mathrm{b}}$ Obesity was defined as a body mass index (BMI) of $\geqslant 30 \mathrm{~kg} \mathrm{~m}^{-2}$ ${ }^{\mathrm{c}}$ For practical purposes, a participant was considered as having hypothyroidism if he/she had a diagnostic of hypothyroidism by his/her primary care provider and was being treated with a stable dose of levothyroxine during the trial, which makes it is likely that some cases of hyperthyrotropinemia may have been included in this group. 'Sleep apnea was defined here by signs and symptoms suggestive of sleep apnea as reported by the caregivers. ${ }^{\text {e}} \mathrm{Fisher}$ 's exact test was used, as cell sizes are $<5$. 'DAS-II Ability score is a Rasch model weighted intermediate score used to convert raw scores to T-scores when different sets of items are administered to patients. It is not equivalent to a standard score. DAS-II does not have norms for patients above 17 years of age. PPVT-III standard scores are provided here for the purpose of describing the sample with regard to level of cognitive functioning. PPVT-III raw scores were used in all other analyses. 
Table 3 Neuropsychological and adaptive functioning measures: pre/post difference scores, $90 \% \mathrm{Cl}$ and $P$-value for the relevant contrast effect in MIXED (one tailed)

\begin{tabular}{|c|c|c|c|c|c|}
\hline \multirow[t]{2}{*}{ Measure } & \multicolumn{2}{|l|}{ Memantine } & \multicolumn{2}{|l|}{ Placebo } & \multirow[t]{2}{*}{ P-value } \\
\hline & Pre/post difference score & $90 \% \mathrm{Cl}$ & Pre/post difference score & $90 \% \mathrm{Cl}$ & \\
\hline \multicolumn{6}{|l|}{ Cognitive and adaptive measures } \\
\hline DAS-II Matrices & 3.39 & -0.47 to 7.25 & -0.32 & -4.07 to 3.44 & 0.126 \\
\hline SIB-R Broad independence & 5.94 & 2.02 to 9.85 & 4.88 & 1.21 to 8.55 & 0.371 \\
\hline \multicolumn{6}{|l|}{ Hippocampal measures } \\
\hline CANTAB PAL Stages & 0.56 & -0.07 to 1.04 & 0 & -0.48 to 0.48 & 0.088 \\
\hline CANTAB PAL First Trial & 0.39 & -1.05 to 1.83 & 0.68 & -0.72 to 2.09 & 0.403 \\
\hline CANTAB PRM & -0.22 & -1.95 to 1.50 & -0.84 & -2.52 to 0.83 & 0.333 \\
\hline CVLT Free Recall Total & 5.84 & 3.47 to 8.21 & 2.53 & 0.33 to 4.72 & $0.046^{*}$ \\
\hline CVLT Free Recall Discrimination & 0.64 & 0.29 to 0.98 & 0.38 & 0.06 to 0.70 & 0.183 \\
\hline RBMT Composite & 1.3 & -0.56 to 3.16 & 0.79 & -0.98 to 2.56 & 0.371 \\
\hline \multicolumn{6}{|l|}{ Pre-frontal measures } \\
\hline NEPSY Verbal Fluency & 0.78 & -1.18 to 2.74 & 0.21 & -1.69 to 2.12 & 0.364 \\
\hline CANTAB SWM Strategy & -0.06 & -1.70 to 1.59 & -1.47 & -3.07 to 0.12 & 0.151 \\
\hline CANTAB SWM Between & -3.33 & -8.84 to 2.17 & 0.32 & -5.05 to 5.68 & 0.214 \\
\hline \multicolumn{6}{|l|}{ Language measures } \\
\hline PPVT-III & 0.72 & -4.02 to 5.46 & -1.42 & -6.03 to 3.19 & 0.294 \\
\hline TROG-II & 2.33 & -0.46 to 5.13 & -0.42 & -3.14 to 2.30 & 0.121 \\
\hline DAS-II Recall of Digits & 5.39 & -3.88 to 14.66 & -7.47 & -16.50 to 1.55 & 0.051 \\
\hline
\end{tabular}

Abbreviations: CANTAB, Cambridge Neuropsychological Test Automated Battery; CI, confidence interval; CVLT-II, California Verbal Learning Test-II; DAS-II, Differential Ability Scales-II; NEPSY, Developmental Neuropsychological Assessment; PAL, Paired Associate Learning; PPVT-III, Peabody Picture Vocabulary Test3rd edition; PRM, Pattern Recognition Memory; RBMT, Rivermead Behavioral Memory Test-Children's version; SIB-R, Scales of Independent Behavior-Revised; SWM, Spatial Working Memory; TROG-II, Test of Reception of Grammar-2nd edition.

*Measures for which we were able to detect treatment effects with $P$-values $<0.05$. Ability score is reported for DAS-II Matrices, and standard score is reported for SIB-R; otherwise, all other means and s.d. are for raw scores. CVLT-II Free Recall Total and CVLT-II Free Recall Discrimination refers to totals over the five learning trials. RBMT Composite is composite of Route Learning, Message, and Belonging from the RBMT Behavioral Memory Test. NEPSY Verbal Fluency comprises 'Food' and 'Drink' semantic categories only. DAS-II only tests forward digits on Recall of Digits subtest.

performed are depicted in graph form in Figure 2a). Finally, a contrast effect bordering statistical significance was found for the DAS-II Recall of Digits score $(\mathrm{F}(1,34)=2.83, P=0.051)$. The memantine pre/post difference score was $5.39(90 \%$ $\mathrm{Cl}=-3.88$ to 14.66$)$, whereas the pre/post difference score for the placebo group was $-7.47(90 \% \mathrm{Cl}=-16.50$ to 1.55$)$. No other primary or secondary memory outcome measures were significant (all $P$-values $>0.10$ ).

As the sample size of this pilot study was small, power to detect group differences was modest. In order to understand further the possibility for significant improvement in cognitive functioning as a result of treatment with memantine, effect sizes for the primary and secondary outcome measures were computed using Cohen's $d$. The latter was performed on the group means of the differences between baseline and 16week testing scores for each of the dependent variables. In the literature, a Cohen's $d$ value of 0.2 is typically considered a small effect, whereas a value of 0.5 is considered a moderate effect. As can be seen in Figure $2 b$, various outcome measures had Cohen's $d$ values approaching a moderate effect size (>0.30), and the CVLT-II Free Recall Total Score as well as the DAS-II Recall of Digits score had Cohen's $d$ values of $>0.5$.

Finally, the relations among the various outcome measures were explored, including their association with age and gender. These analyses were only performed in the entire sample, because of the small sample size, but were done for both the baseline scores and for the scores obtained at 16 weeks. Initially, Pearson's $r$ was computed for each dependent variable with age. There were numerous significant correlations at both time points, with the strongest associa- tions observed between age and the language and adaptive functioning scores (all $P$-values $<0.05$, two tailed). At baseline, there was also a significant association between age and PRM raw score $(r=0.33, P=0.04)$, and between age and Spatial Working Memory (SWM)-Strategy $(r=0.38$, $P=0.019)$. At 16 weeks, age was also significantly correlated with CVLT-II Total Free Recall, CVLT-II Free Recall Discriminability and PRM ( $r$ values ranging from 0.33 to 0.36 , all $P$-values $<0.05)$. These associations with age were expected, as many of the dependent variables were raw scores and there was a wide age range represented in the sample.

Partial correlations were then computed among the outcome variables, as well as between the outcome variables and gender, with age as a covariate. At baseline, gender was correlated with the two primary outcome measures of memory ( $r=0.48$ with PRM and $r=0.35$ with PAL, both $P<0.05$ ). Female gender was associated with better scores on these measures. Gender was also significantly correlated with Recall of Digits and SWM-Strategy ( $r$ values of 0.36 and 0.39 , respectively, $P$-values $<0.05$ ). Intercorrelations among tests measuring the same cognitive domain (that is, memory, language, prefrontal function, adaptive functioning) were all significant $(P$-values $<0.01$ or better).

Partial correlations computed with the data obtained at 16 weeks, again covarying age, showed a very similar pattern. Gender was significantly correlated with two memory outcome measures: the RBMT composite and the PAL scores ( $r$ of 0.33 and 0.34 , respectively, both $P$-values $<0.05$ ). Gender was also significantly correlated with Recall of Digits $(r=0.39, P<0.05)$. As at baseline, all tests within a given 

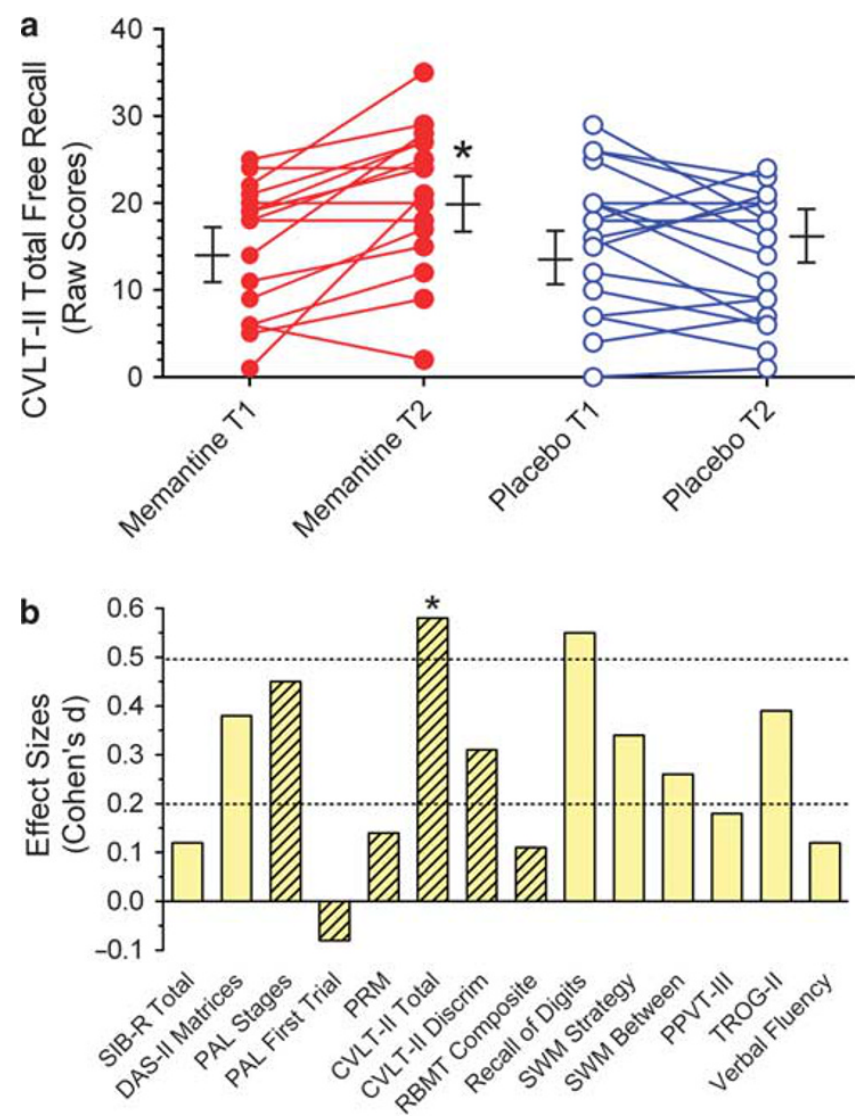

Figure 2 (a) Before and after, dot plot representations of individual CVLT-Il total free recall raw scores and the magnitude of change (denoted by straight lines connecting the dots representing individual measurement values) from the time of the baseline neuropsychological testing, before exposure to the study medication (T1) to the end of the 16-week trial (T2). The graphs in red (filled circles) and blue (open circles) represent the memantine and placebo groups, respectively. The symbol (*' denotes the $P$-value of $<0.05$ for the difference between T1 and T2 for the memantine group compared with the placebo group using MIXED models. Error bars represent mean and $90 \%$ confidence intervals. Note that the CVLT-II total free recall raw scores from 11 patients showed increases from T1 to T2, whereas this only happened for scores from 6 patients in the placebo group. Also, increases in score larger than 10 points only happened for the memantine group. (b) Bar graph representing the effect size (Cohen's $d$ ) for neuropsychological and adaptive functioning variables assessed in this study. The symbol '*' denote a treatment effect with $P$-values $<0.05$. The hashed bars represent primary measures or secondary measures associated with the primary hypothesis, whereas the solid bars represent secondary discriminant measures. The dashed horizontal lines represent the 0.2 level (at which an effect size is typically considered small), and the 0.5 level (moderate effect size). CVLT-II, California Verbal Learning Test-II; DAS-II, Differential Ability Scales-II; PAL, Paired Associate Learning; PPVT-III, Peabody Picture Vocabulary Test-3rd edition; RBMT, Rivermead Behavioral Memory Test-Children's version; SIB-R, Scales of Independent Behavior-Revised; SWM, Spatial Working Memory; TROG-II, Test of Reception of Grammar-2nd edition.

cognitive domain were significantly correlated with one another (all $P$-values $<0.05$ ). The small sample size precluded analyzing whether the significant memantine effects reported in the main analyses interacted with gender.

Safety and tolerability of memantine. There were no laboratory adverse events and no serious adverse events. In total, 4 adverse events were reported in the memantine group by either the caregiver or the participant: 2 of 20 participants (10\%) reported increased anxiety, in which 1 case was deemed serious enough by a parent who requested to have the treatment discontinued at week 4 , the other participant received treatment for 16 weeks but was unable to produce consistent responses at the follow-up neuropsychological assessment; one participant (5\%) complained of transient dizziness that lasted for 2 to 3 days during the first 8 weeks of medication, the condition resolved spontaneously, and the participant underwent the complete 16-week treatment without further complaints; and the parent of one participant $(5 \%)$ complained of echolalia. In the placebo group, one participant (5\%) displayed hair loss, which was followed up by a dermatologist at the Children's Hospital Colorado who made the diagnosis of androgenic alopecia. Except for the increased anxiety in the two participants who received memantine treatment, none of the reported adverse events were judged clinically significant.

\section{Discussion}

We report the results of a small randomized, double-blind, placebo-controlled trial to investigate the effects of the drug memantine on cognitive outcome measures in young adults with DS. We used a carefully selected set of neuropsychological outcomes to test our primary hypothesis that memantine might improve hippocampal-dependent function in individuals with DS. This hypothesis was based on preclinical 
studies in a mouse model of DS. We found a significant effect of 16 weeks of memantine treatment on one secondary memory outcome measure associated with the primary hypothesis, the CVLT-II. Additionally, we found effects with $P$-values $<0.10$ for one of the primary outcome measures, the PAL Stages, and for one of the secondary/discriminant outcome measures, the DAS-II Recall of Digits. These findings should warrant further investigation with the use of a larger sample size. Memantine therapy was well tolerated in our participant cohort, with only mild and infrequent adverse events noted. Given our findings, it would be premature to make any recommendations on the clinical usefulness of memantine as a therapeutic agent for the amelioration of the cognitive deficits associated with DS. This trial now joins a group of very few placebo-controlled trials to be performed on individuals with DS and was a necessary step toward the establishment of a bridge between the preclinical work with animal models of DS and the much more ambitious project of investigating various pharmacotherapies to improve the quality of life of individuals with DS.

Ultimately, the most clinically relevant measures for the evaluation of the efficacy of medications directed at enhancing the cognitive capabilities of individuals with intellectual disability of any cause are those assessing adaptive and daily living skills. These are the practical, everyday skills that affect independence and social competence. However, these skills also take several years to mature, even in the population of typically developing individuals. Hence, it would have been unrealistic to expect any funding agency or pharmaceutical company to finance the implementation of the large-scale and prolonged study that would be required to demonstrate efficacy in adaptive and daily living skills at an exploratory stage of investigation (see Costa ${ }^{24}$ for a recent review on this subject). Accordingly, the design of the present study was based on modest, but realistic goals.

The finding of improved CVLT-II scores in the group of participants with DS treated with memantine in the present study merits further consideration. The CVLT-II is one of the five most common assessment instruments used by clinical neuropsychologists in North America. ${ }^{25}$ The construct validity of the CVLT-II as a measure of episodic verbal learning and memory has garnered considerable support in the neuropsychological literature ${ }^{26,27}$ and it has been used to assess the levels of severity and recovery in neurological disorders including $A D$, tumors, traumatic brain injury, stroke and epilepsy affecting the medial temporal lobe $26,28-31$ and neuropsychiatric afflictions such as posttraumatic stress disorder and chronic depression. ${ }^{32-34}$ The CVLT-II is commonly used to evaluate verbal memory, and consists of the presentation of a word list that is longer than the average working memory span. ${ }^{34}$ Correct encoding, storage and consolidation are needed for long-term memory, and these processes have been shown to be heavily dependent on the function of hippocampus/temporal lobes. In healthy, typically developing individuals, it is generally assumed that successful verbal learning of a supraspan word list also depends on the development of a strategy, and should therefore rely on the frontal lobes as well. Finally, attention deficits or interference may also contribute to disturbed recall. In pathologic conditions, such as in amnesia or memory impairment, subjects are unlikely to develop alternative learning strategies, which make the test even more dependent on the functioning of hippocampus/medial temporal lobes. Indeed, there is general agreement on the role of the medial temporal lobes in episodic/long-term consolidation, and most researchers agree that verbal memory generally depends more on leftside structures. ${ }^{35}$ Although it would have been interesting to characterize serial position effects and subjective clustering strategies associated with the CVLT-II performance by individuals with DS in the present study, the use of the short form of the CVLT-II (which has 9 items instead of 16) has limited the potential usefulness of such analysis. The finding of a borderline significant effect on the DAS-II Recall of Digits is also intriguing and deserves future investigation, because of this measure's dependence on prefrontal cortex function.

In spite of its broad use in clinical and experimental neuropsychology, in the present work, we chose not to describe the CVLT-II as a primary measure, but instead as a 'secondary measure associated with the primary hypothesis'. This was consciously done because, in the strictest sense of the term, a primary measure of a clinical trial should be one from which the a priori power calculations are performed for the estimation of the necessary sample size to detect significance at a pre-established level of power. The only two measures that fit this description in the present trial were the PAL and PRM. Previously published mean and s.d. data were already available from the work of Pennington et al. ${ }^{7}$ at the time this study was conceived. However, no CVLT-II assessments in individuals with DS were available in the literature. Therefore, we were unable to make any statistical estimation of sample sizes based on expected effect sizes. Now, the data showed here will allow us and others to design trials in which the CVLT-II will feature as the (or at least one of the) primary measure(s).

As the fields of pharmacotherapeutics for DS and other defined central nervous system disorders associated with cognitive and intellectual disabilities move forward, an important question to ponder is the translatability of behavioral measures obtained in an animal model (generally a rodent species) into neuropsychological measures from human studies. In the original collaboration between the research teams led by Drs Bruce Pennington and Lynn Nadel, ${ }^{7}$ their emphasis on tests with a significant visuospatial memory component, such as the PAL and PRM, was based on the premise that these types of tests were more likely to have similar construct validity between mouse and human studies. However, here the most promising measure of hippocampusdependent function was the CVLT-II, which obviously cannot be assessed in mice. It is not unreasonable to argue that, evolutionarily, verbal episodic memory has acquired a disproportionate importance in the human medial temporal lobe, compared with visuospatial memory and the formation of cognitive maps.

In addition to its small sample size, short duration of the treatment and the use of multiple statistical comparisons without adjusting the $P$-value, this study has a few other limitations. For example, participant selection was biased toward a subgroup of young adults with DS who were generally healthy, verbal and with no, or very few, behavioral issues. One of the consequences of this bias was the 
production of an $\sim 2: 1$ imbalance in the female/male ratio in our sample. This can be explained by the observation that, in average, adolescent and young adult women with DS have been reported to function at a higher cognitive level and display less maladaptive behaviors and psychiatric disorders than their male counterparts. ${ }^{36,37}$ Also, although extreme care was taken in the selection of participants and caregivers, we acknowledge the very small, but real, possibility that a few caregivers might have removed the study medication from the bottles without giving them to the participants.

In selecting the age range, our goal was to avoid the potential confound of neurodevelopment during the 16-week time window between the baseline neuropsychological evaluation and the posttreatment assessment, while also minimizing any superimposing effect of neurodegeneration on these measures. The pitfalls represented by recruiting participants of advanced age and concomitant health issues were seen recently in the study by Hanney et al., ${ }^{38}$ named 'Memantine for dementia in adults older than 40 years with Down's syndrome' (MEADOWS). This study was a randomized, double-blind, placebo-controlled trial to assess safety and efficacy of memantine on the treatment of cognitive decline associated with dementia in individuals with DS. Although 1-year-long treatment with memantine was well tolerated in this participant cohort, efficacy data were completely negative. The design of the MEADOWS trial preceded many important recent findings in the field of DS research; therefore, it did not benefit from newly available information. For example, neuropsychological assessments of hippocampus-dependent measures were not included in the protocol. Additionally, it is plausible to assume that, by the time treatment began, various irreversible pathological cellular cascades were already triggered and the disease process might have reached a point of no return (see Costa ${ }^{39}$ for a more complete set of comments on the MEADOWS trial). Therefore, an investigation of the potential neuroprotective role of memantine in young, healthy adults with DS cannot be discarded in light of the present evidence.

Because of the novel nature of some of the findings in the present study, it would be prudent to proceed with caution and our results should be reproduced in a larger sample of participants before an even larger, phase III multicenter trial can be planned. Although memantine therapy has proven safe and well tolerated here and in the MEADOWS trial, given the expression of increased anxiety in some participants in both trials, a dose-finding trial may also be warranted. Eventually, we also would like to design future trials specifically to include younger, school-age children, similar to what has been done recently by Kishnani et al., ${ }^{40}$ where these authors investigated the potential usefulness of the anticholinesterase drug donepezil for treatment of cognitive dysfunction in children with DS aged 10-17 years. At present, however, very little clinical (or even preclinical) safety data on memantine are available for pediatric participants. Finally, it would be important to explore the usefulness of electrophysiological, imaging and/or biochemical biomarkers in future studies. Such biomarkers would not only provide potential surrogate end points in addition to neuropsychological measures, but also might allow for a more inclusive recruitment in future studies. One could argue, for example, that individuals who are nonverbal and/or display significant behavioral issues are exactly those who might benefit the most from therapies directed at enhancing cognitive abilities in individuals with DS.

\section{Conflict of interest}

The senior author (ACSC) is the Principal Investigator of an Investigator-Initiated Grant from the Forest Research Institute used to fund this study. The other authors declare no conflict of interest.

Acknowledgements. We thank the study participants and their parents, the Mile High Down Syndrome Association, the Denver Adult Down Syndrome Clinic and the Colorado Springs Down Syndrome Association for their help with participant recruitment. None of the participants received any form of compensation for their contributions. We also thank Diane Fairclough and Bruce Pennington. This trial was funded by Forest Research Institute Investigator Initiated Grant NAM-58. ACSC was also supported in part by NIH Grant RO1-HD056235, the Linda Crnic Institute and the Coleman Institute for Cognitive Disabilities. The funding sources had no role in the design and conduct of the study; in the collection, analysis and interpretation of the data; or in the preparation, review or approval of the manuscript.

1. Lejeune J, Turpin R, Gautier M. Le mongolism: premier exemple d'aberration autosomique humaine. Ann Genet 1959; 1: 1-49.

2. Patterson D, Costa AC. Down syndrome and genetics - a case of linked histories. Nat Rev Genet 2005; 6: 137-147.

3. Parker SE, Mai CT, Canfield MA, Rickard R, Wang Y, Meyer RE et al. National Birth Defects Prevention Network. Updated National Birth Prevalence estimates for selected birth defects in the United States, 2004-2006. Birth Defects Res A Clin Mol Teratol 2010; 88: 1008-1016

4. Pueschel SM, Hopmann MR. Speech and language abilities of children with Down syndrome: a parents's perspective. In: Kaiser AP, Gray DB (eds). Enhancing Children's Communication: Research Foundations for Intervention Communication and Language Intervention Series, vol. 2. P.H. Brookes: Baltimore, 1993 pp 335-362.

5. Carr J. Stability and change in cognitive ability over the life span: a comparison of populations with and without Down's syndrome. J Intellect Disabil Res 2005; 49: 915-928.

6. Turner S, Alborz A, Gayle V. Predictors of academic attainments of young people with Down's syndrome. J Intellect Disabil Res 2008; 52: 380-392.

7. Pennington BF, Moon J, Edgin J, Stedron J, Nadel L. The neuropsychology of Down syndrome: evidence for hippocampal dysfunction. Child Dev 2003; 74: 75-93.

8. Silverman W. Down syndrome: cognitive phenotype. Ment Retard Dev Disabil Res Rev 2007; 13: 228-236.

9. Lott IT, Dierssen M. Cognitive deficits and associated neurological complications in individuals with Down's syndrome. Lancet Neurol 2010; 9: 623-633.

10. Edgin JO, Mason GM, Allman MJ, Capone GT, Deleon I, Maslen C et al. Development and validation of the Arizona Cognitive Test Battery for Down syndrome. $J$ Neurodev Disord 2010; 2: 149-164.

11. Edgin JO, Kumar A, Spano G, Nadel L. Neuropsychological effects of second language exposure in Down syndrome. J Intellect Disabil Res 2011; 55: 351-356.

12. Roizen NJ, Patterson D. Down's syndrome. Lancet 2003; 361: 1281-1289.

13. Zigman WB, Lott IT. Alzheimer's disease in Down syndrome: neurobiology and risk. Ment Retard Dev Disabil Res Rev 2007; 13: 237-246.

14. Costa AC. An assessment of the vestibulo-ocular reflex (VOR) in persons with Down syndrome. Exp Brain Res 2011; 214: 199-213.

15. Costa AC. An assessment of optokinetic nystagmus (OKN) in persons with Down syndrome. Exp Brain Res 2011; 214: 381-391.

16. Costa AC, Scott-McKean JJ, Stasko MR. Acute injections of the NMDA receptor antagonist memantine rescue performance deficits of the Ts65Dn mouse model of Down syndrome on a fear conditioning test. Neuropsychopharmacology 2008; 33: 1624-1632.

17. Lockrow J, Boger H, Bimonte-Nelson H, Granholm AC. Effects of long-term memantine on memory and neuropathology in Ts65Dn mice, a model for Down syndrome. Behav Brain Res 2011; 221: 610-622.

18. Rueda N, Llorens-Martin M, Florez J, Valdizan E, Banerjee P, Trejo JL et al. Memantine normalizes several phenotypic features in the Ts65Dn mouse model of Down syndrome. $J$ Alzheimers Dis 2010; 21: 277-290.

19. Scott-McKean JJ, Costa AC. Exaggerated NMDA mediated LTD in a mouse model of Down syndrome and pharmacological rescuing by memantine. Learn Mem 2011; 18: 774-778.

20. McShane R, Areosa Sastre A, Minakaran N. Memantine for dementia. Cochrane Database Syst Rev 2006; : CD003154.

21. Herrmann N, Li A, Lanctot K. Memantine in dementia: a review of the current evidence. Expert Opin Pharmacother 2011; 12: 787-800. 
22. Lo D, Grossberg GT. Use of memantine for the treatment of dementia. Expert Rev Neurother 2011; 11: 1359-1370.

23. Hodapp AF, Gerken KC. Correlations between scores for Peabody Picture Vocabulary Test-III and the Wechsler Intelligence Scale for Children-III. Psychol Rep 1999; 84: $1139-1142$.

24. Costa AC. On the promise of pharmacotherapies targeted at cognitive and neurodegenerative components of Down syndrome. Dev Neurosci 2011; 33: 414-427.

25. Rabin LA, Barr WB, Burton LA. Assessment practices of clinical neuropsychologists in the United States and Canada: a survey of INS, NAN, and APA Division 40 members. Arch Clin Neuropsychol 2005; 20: 33-65.

26. Alexander MP, Stuss DT, Fansabedian N. California Verbal Learning Test: performance by patients with focal frontal and non-frontal lesions. Brain 2003; 126: 1493-1503.

27. Donders J. A confirmatory factor analysis of the California Verbal Learning Test-Second Edition (CVLT-II) in the standardization sample. Assessment 2008; 15: 123-131.

28. Reeves CB, Palmer SL, Reddick WE, Merchant TE, Buchanan GM, Gajjar A et al. Attention and memory functioning among pediatric patients with medulloblastoma. J Pediatr Psychol 2006; 31: 272-280.

29. Cherney LR, Halper AS. Performance on the California Verbal Learning Test following right hemisphere stroke: a longitudinal study. Top Stroke Rehabil 2007; 14: 21-25.

30. DeJong J, Donders J. A confirmatory factor analysis of the California Verbal Learning TestSecond Edition (CVLT-II) in a traumatic brain injury sample. Assessment 2009; 16: 328-336.

31. Lekeu F, Magis D, Marique P, Delbeuck X, Bechet S, Guillaume B et al. The California Verbal Learning Test and other standard clinical neuropsychological tests to predict conversion from mild memory impairment to dementia. J Clin Exp Neuropsychol 2010; 32 164-173.

32. Quigg M, Broshek DK, Barbaro NM, Ward MM, Laxer KD, Yan G et al. Neuropsychological outcomes after Gamma Knife radiosurgery for mesial temporal lobe epilepsy: a prospective multicenter study. Epilepsia 2011; 52: 909-916.
33. Mesholam-Gately RI, Giuliano AJ, Zillmer EA, Barakat LP, Kumar A, Gur RC et al. Verbal learning and memory in older adults with minor and major depression. Arch Clin Neuropsychol 2012; 27: 196-207.

34. Johnsen GE, Asbjornsen AE. Consistent impaired verbal memory in PTSD: a metaanalysis. J Affect Disord 2008; 111: 74-82.

35. Albuquerque L, Loureiro $\mathrm{C}$, Martins IP. Effect of lesion site on serial position during list learning: a study with the CVLT. Int J Neurosci 2008; 118: 917-933.

36. Lund J. Psychiatric aspects of Down's syndrome. Acta Psychiatr Scand 1988; 78: 369-374

37. Capone GT, Grados MA, Kaufmann WE, Bernad-Ripoll S, Jewell A. Down syndrome and comorbid autism-spectrum disorder: characterization using the aberrant behavior checklist. Am J Med Genet A 2005; 134: 373-380.

38. Hanney M, Prasher V, Williams N, Jones EL, Aarsland D, Corbett A et al. Memantine for dementia in adults older than 40 years with Down's syndrome (MEADOWS): a randomised, double-blind, placebo-controlled trial. Lancet 2012; 379: 528-536.

39. Costa AC. Alzheimer disease: treatment of Alzheimer disease in Down syndrome. Nat Rev Neurol 2012; 8: 182-184.

40. Kishnani PS, Heller JH, Spiridigliozzi GA, Lott I, Escobar L, Richardson S et al. Donepezil for treatment of cognitive dysfunction in children with Down syndrome aged 10-17. Am J Med Genet A 2010; 152A: 3028-3035.

Translational Psychiatry is an open-access journal published by Nature Publishing Group. This work is licensed under the Creative Commons Attribution-Noncommercial-No Derivative Works 3.0 Unported License. To view a copy of this license, visit http://creativecommons.org/licenses/by-nc-nd/3.0/

Supplementary Information accompanies the paper on the Translational Psychiatry website (http://www.nature.com/tp) 